\title{
Effective Elastic Properties for Lower Limb Soft Tissues from Manual Indentation Experiment
}

\author{
Yongping Zheng, Member, IEEE, and Arthur F. T. Mak, Member, IEEE
}

\begin{abstract}
Quantitative assessment of the biomechanical properties of limb soft tissues has become more important during the last decade because of the introduction of computer-aided design and computer-aided manufacturing (CAD/CAM) and finite element analysis to the prosthetic socket design. Because of the lack of a clinically easy-to-use apparatus, the site and posture dependences of the material properties of lower limb soft tissues have not been fully reported in the literature. In this study, an ultrasound indentation system with a pen-size handheld probe developed earlier by the authors was used to obtain the indentation responses of lower limb soft tissues. Indentation tests were conducted on normal young subjects with four females and four males at four sites with three body postures. A linear elastic indentation solution was used to extract the effective Young's modulus from the indentation responses. The determined modulus ranged from 10.4 to $89.2 \mathrm{kPa}$ for the soft tissues tested. These results were in a similar range as those reported in the literature. The thickness of the lower limb soft tissues varied slightly with body posture changes. The Young's modulus determined was demonstrated to be significantly dependent on site, posture, subject and gender. The overall mean modulus of male subjects was $40 \%$ larger than that of female subjects. No significant correlation was established between the effective Young's modulus and the thickness of entire soft tissue layers.
\end{abstract}

\section{INTRODUCTION}

$\mathbf{T}$ HE human musculoskeletal system is entirely covered by layers of soft tissues, which are comprised of skin, fat, muscle, embedded blood vessels, tendon, and ligament. The material properties of soft tissues in the musculoskeletal system have usually been measured along their long axis or line of action, i.e., for tendons, ligaments and muscles, and for skin, in the plane of its surface. However, at the body support interfaces, such as the buttock tissues interfacing with the seat, the residual limb tissues interacting with the prosthetic socket, and the plantar tissues of the foot interacting with the in-sole/ground, significant loads normal to the skin surface are transmitted via the skin to the underlying tissues. Biomechanical assessment of skin and the underlying soft tissues normal to the body surface is relevant to the designs of these body support interfaces. These issues are relevant to many clinical rehabilitation problems, such as special seatings for the spinal cord injured, special orthotics for diabetic feet, and custom prosthetic sockets for amputees.

Manuscript received April 23, 1997; revised April 23, 1998 and June 3, 1999. This work was supported by the Research Grant Council of Hong Kong.

The authors are with the Rehabilitation Engineering Center, The Hong Kong Polytechnic University, Kowloon, Hong Kong (e-mail: rcafmak@polyu.edu.hk).

Publisher Item Identifier S 1063-6528(99)07103-7.
Palpation is often employed in clinical evaluation of some pathological conditions of soft tissues, such as subcutaneous oedema. An indentation apparatus was developed by Schade et al. to study the changes of creep properties of skin and subcutaneous tissues of limbs in oedematous conditions [24]. Similar investigations using different indentation apparati were also reported recently [6], [18]. Investigations in this general area have reported the dependence of these biomechanical properties on site, subject, age, and gender [1], [5], [9], [13], [14], [17], [20], [36]. Testing sites on lower limbs and forearms were usually selected in those investigations. Computer-aided design and computer-aided manufacturing (CAD/CAM) techniques and finite element (FE) analysis have been introduced into the design of prosthetic sockets and cushions [2], [4], [10], [15], [23]. Determination of quantitative biomechanical properties in residual limb tissues in compression has become important so as to provide inputs to these advance design tools. If the individual profiles of thickness and material properties of the residual limb tissues can be incorporated as additional inputs to the CAD software in addition to the residual limb geometry which is the only quantitative input to the design process currently, socket design can then be carried out in a more objective manner. Compared with the conventional methods of socket design which strongly depend on the experience of prosthetists, this new approach will help to make knowledge accumulation easier and teaching-learning more precise for the socket fabrication. However, quantitative material properties of soft tissues have not been involved in the current CAD/CAM socket design system. One of the main reasons may be the lack of an easy-to-use approach for quantitative biomechanical assessment of soft tissues in vivo. In addition, FE analysis has been adopted to study the interface stress between the surface of the residual limb tissue and the socket during the last decade. It is anticipated that FE analysis may effectively predict stress distributions for a proposed socket design, thus offering the opportunities to evaluate the design before prosthetic fabrication. Individual biomechanical properties and thickness of the residual limb tissues have been recognized as important inputs to the residual limb-socket FE modeling [22], [25]-[27], [30].

If these approaches can ultimately be introduced to the clinical practice, an easy-to-use approach for individual biomechanical assessment of residual limb tissues will definitely be needed. A number of apparati have been developed for this purpose during the last decade [19]-[21], [26]-[29]. However, some of these apparati were designed as research tools and 


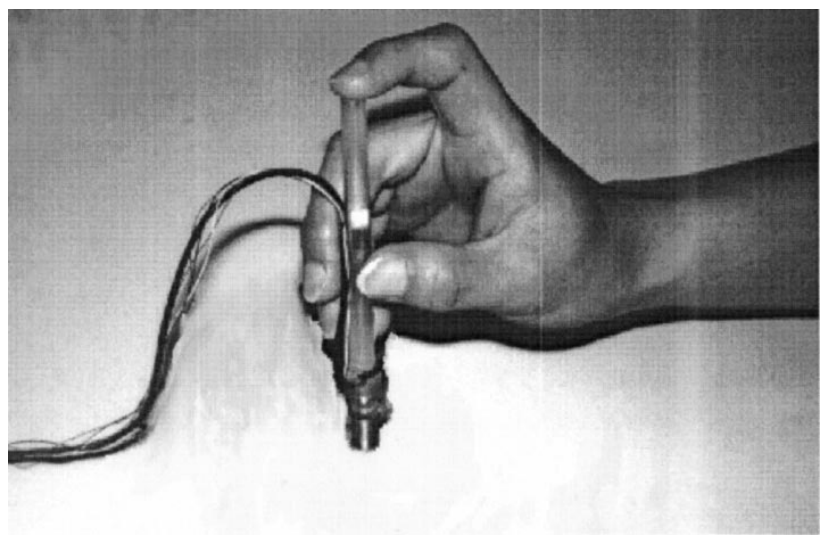

Fig. 1. The operation of the pen-size indentation testing probe.

might not readily render themselves as user-friendly clinical tools.

The technique of using ultrasound to measure tissue thickness of oral mucosa has been proposed before [3], [16]. This technique has also been used in association with a cantilever loading device to investigate the age-dependent creep response of skin and subcutaneous tissues at a site over the anteromedial aspect of tibia [17]. However, the experimental set up of those devices have not been detailed in that paper. A pen-size ultrasound indentation probe has been earlier developed by the authors [31]-[35]. Load-indentation response as well as tissue thickness can be obtained quickly by using the new apparatus. This made it possible to perform more extensive investigations on biomechanical assessment of limb soft tissues under various conditions. The objective of this paper is to investigate the effective Young's modulus and tissue thickness of the lower limb soft tissues at different sites and with different body postures. The correlations of the effective Young's modulus of these soft tissues with gender as well as with tissue thickness were also addressed in this paper.

\section{Methodology}

\section{A. Ultrasound Indentation Apparatus}

The ultrasound indentation apparatus was comprised of a pen-size, hand-held indentation probe (see Fig. 1). An ultrasound transducer with a diameter of $9 \mathrm{~mm}$ was at the tip of the probe and also served as the indentor. The thickness and deformation of the soft tissue layer were determined from the ultrasound echo signal. A compressive load cell was connected in series with the ultrasound transducer to record the corresponding force response. A schematic of the indentation system is shown in Fig. 2. Software was developed to display in real time the ultrasound reflection signals, deformation transient and force transient on the computer monitor. All the data could be recorded in a file for further off-line study, and the whole procedure could be played back continuously or in incremental time steps. A pair of cursors within the ultrasound wave train could be set for autotracking the echo peaks during the indentation procedure. The ultrasound echo wave reflected from the soft tissue-bone interface would move as the soft

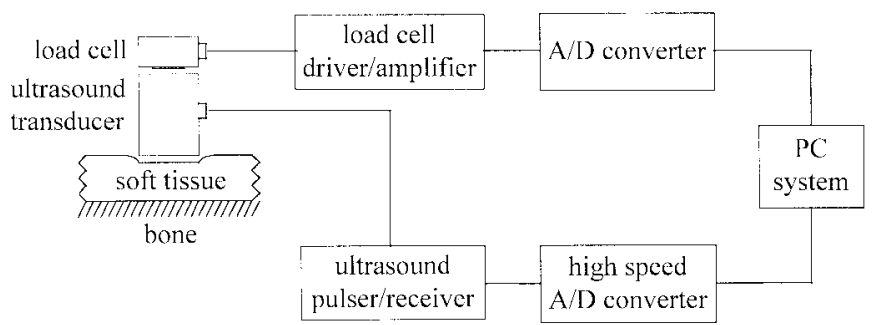

Fig. 2. A schematics showing the ultrasound indentation system.

tissue thickness changed during loading and unloading. The algorithm calculated the deflection of the time of flight of the ultrasound echo wave with the reference of the wave peak to determine tissue deformation by assuming the sound velocity in soft tissues was $1540 \mathrm{~m} / \mathrm{s}$ [7].

By increasing the power of the transmitted ultrasound pulse and the gain of the receiving amplifier, ultrasound signals reflected from the soft tissue-bone interface as deep as 15 $\mathrm{cm}$ in the thigh could be detected using the probe. With a Sonix (Springfield, VA) $100 \mathrm{MHz}$ 8-bit analog-to-digital (A/D) converter for digitizing ultrasound signals, the accuracy of the time measurement was $10 \mathrm{~ns}$. The accuracy of the deformation determined by the indentation system was better than $0.02 \mathrm{~mm}$, with the ultrasound frequency above $5 \mathrm{MHz}$ and the amplitude of the ultrasound signal larger than $10 \%$ of the full dynamic range. A 12-bit A/D converter was used to digitize the force signal, and the accuracy of force result was better than 0.003 $\mathrm{N}$ within a $0-10 \mathrm{~N}$ range.

A Hounsfield (Surrey, England) material testing machine was used to validate the ultrasound indentation system. The hand-held probe was placed in line with the load cell of the material testing machine. Force and deformation data were read from the machine by the software via the RS232 port. Similar data were also acquired with the ultrasound indentation system. The validation experiments were performed on a layer of fresh porcine tissue with an initial thickness of $24 \mathrm{~mm}$. The ultrasound echo wave was reflected from the interface between the tissue bottom surface and the container. An ultrasound velocity of $1540 \mathrm{~m} / \mathrm{s}$ was assumed for the entire porcine tissue layer. The probe was first driven to slightly touch the skin surface of the porcine tissue, and all the readouts were reset to zero, then several loading-holding-unloading sequences were imposed manually via the control panel of the material testing machine. The indenting rate imposed varied from 0.5 to $1 \mathrm{~mm} / \mathrm{s}$. The maximum load was approximately $5 \mathrm{~N}$ corresponding to approximately $20 \%$ deformation of the entire tissue layer.

\section{B. Subject Selection}

Four normal young males and four normal young females with age of $28 \pm 2$ participated in the experiments. The mean age of selected females was similar as that of the males. Table I gives the basic information on the eight subjects. The indentation tests were carried out at four sites over the distal femur, proximal tibia and fibula, and popliteal area, respectively. 
TABLE I

Basic Information of the Eight SubJects Participated in the ExPeriments

\begin{tabular}{lrrrrrrrrrr}
\hline Information & YVO & JES & FEN & LIY & TOM & STE & TER & HJY & mean & SD \\
\hline Sex & $\mathrm{F}$ & $\mathrm{F}$ & $\mathrm{F}$ & $\dot{\mathrm{F}}$ & $\mathrm{M}$ & $\mathrm{M}$ & $\mathrm{M}$ & $\mathrm{M}$ & & \\
Age & 27 & 26 & 27 & 31 & 27 & 30 & 26 & 30 & 28.0 & 2.0 \\
Mass $(\mathrm{kg})$ & 50 & 51 & 54 & 47 & 73 & 65 & 60 & 60 & 57.5 & 8.7 \\
Height $(\mathrm{cm})$ & 152 & 158 & 156 & 160 & 165 & 168 & 183 & 166 & 163.5 & 9.5 \\
\hline
\end{tabular}

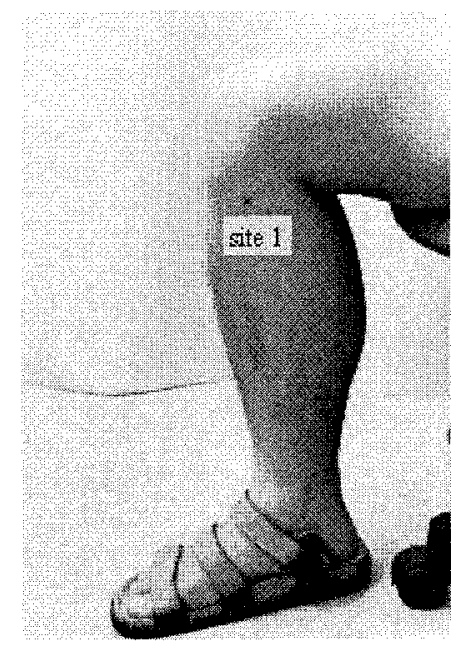

(a)

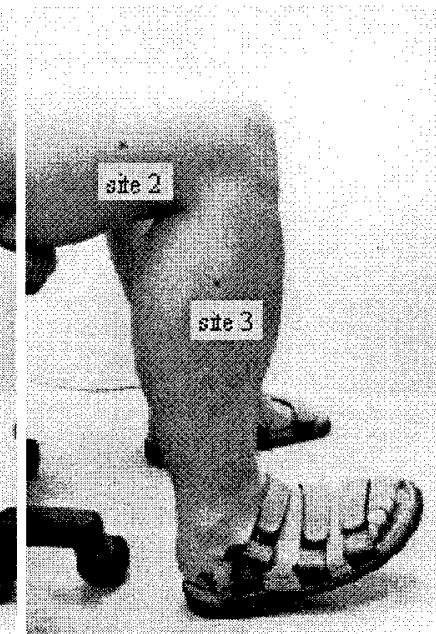

(b)

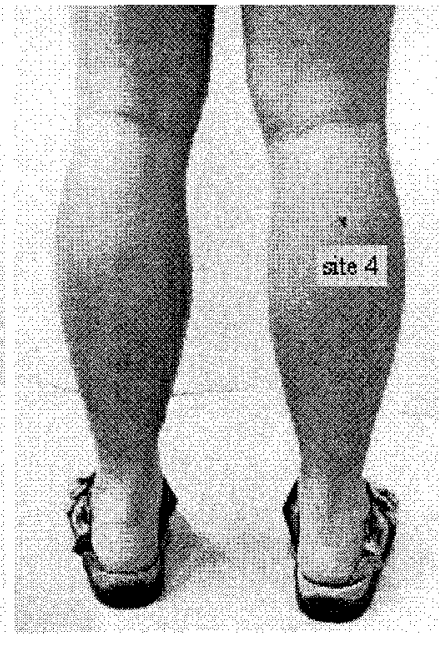

(c)

Fig. 3. The three postures and the four indentation sites over distal femur and proximal tibia and fibula of the right leg. The three postures were (a) sitting with the knee rested at $90^{\circ}$ flexion, (b) posture A but with the foot dorsiflexed, and (c) standing with the knee locked in hyperextension.

\section{Testing Protocol}

Fig. 3 shows the four indentation sites and the three postures selected for this study. The locations of the four sites on the right lower limb were on the soft tissue:

Site 1: over the medial side of the tibia, and $3 \mathrm{~cm}$ from the proximal head of tibia;

Site 2: over the lateral side of the femur, and $10 \mathrm{~cm}$ above from the distal end of femur;

Site 3: over the lateral side of the fibula, and $5 \mathrm{~cm}$ from the proximal head of fibula;

Site 4: over the popliteal area at the same level as site 3.

There were three sites in the region of the proximal tibia and fibula and one site in the region of the distal femur with different soft tissue thickness. These four sites were selected as they are involved in the design of below-knee prosthetic sockets.

Indentation tests were conducted for three body postures, involving different states of muscular contraction around the lower leg. The selected three postures were as follows:

A) sitting with the thigh horizontal and the knee rested at $90^{\circ}$ flexion;

B) posture A but with the foot dorsiflexed;

C) standing with the knee locked in hyperextension.

The subject was asked to sit in a chair with the knee rested at $90^{\circ}$ flexion, and the chair was adjusted to keep the thigh horizontal (posture A). After the tests were completed for posture $A$, the subject was instructed to dorsiflex the foot (posture B), and to maintain the maximal dorsiflexion during a trial. No resistance was applied in the test. In the tests for the third posture, the subject was asked to stand with the knee locked in hyperextension and without additional activation of underlying muscles of the thigh (posture C).

Sitting (posture A) and standing (posture C) are two common human body postures even for amputees, and were selected in this study. The underlying muscles of the four testing sites would be involved during the alternation between these two postures. The typical relationship between biomechanical properties of soft tissues and body postures would be established through the tests with these two postures. The underlying muscles at Site 2 would only be slightly influenced with foot dorsiflexion (i.e., Posture A versus Posture B), when the subject was in the sitting posture. Since Posture B can be readily adopted from Posture A and vice versa with minimal changes in the general body configurations, this would be used to further verify that the activation state of the underlying muscle was the dominant source for the change of biomechanical properties of soft tissues under a testing site.

Indentation testing was manually performed using the pensize probe. Before each test, the probe was used to load and unload the testing site several times to obtain a maximum and stable ultrasound signal reflected from the soft tissue-bone interface. Through many preliminary tests, it was found that tissue preconditioning could be achieved through the same process. A similar result has been reported in the literature [28]. Each test was conducted with a few cycles of loadingunloading sequences with indentation rate manually controlled to be approximately $4 \mathrm{~mm} / \mathrm{s}$. Indentation rate around this 


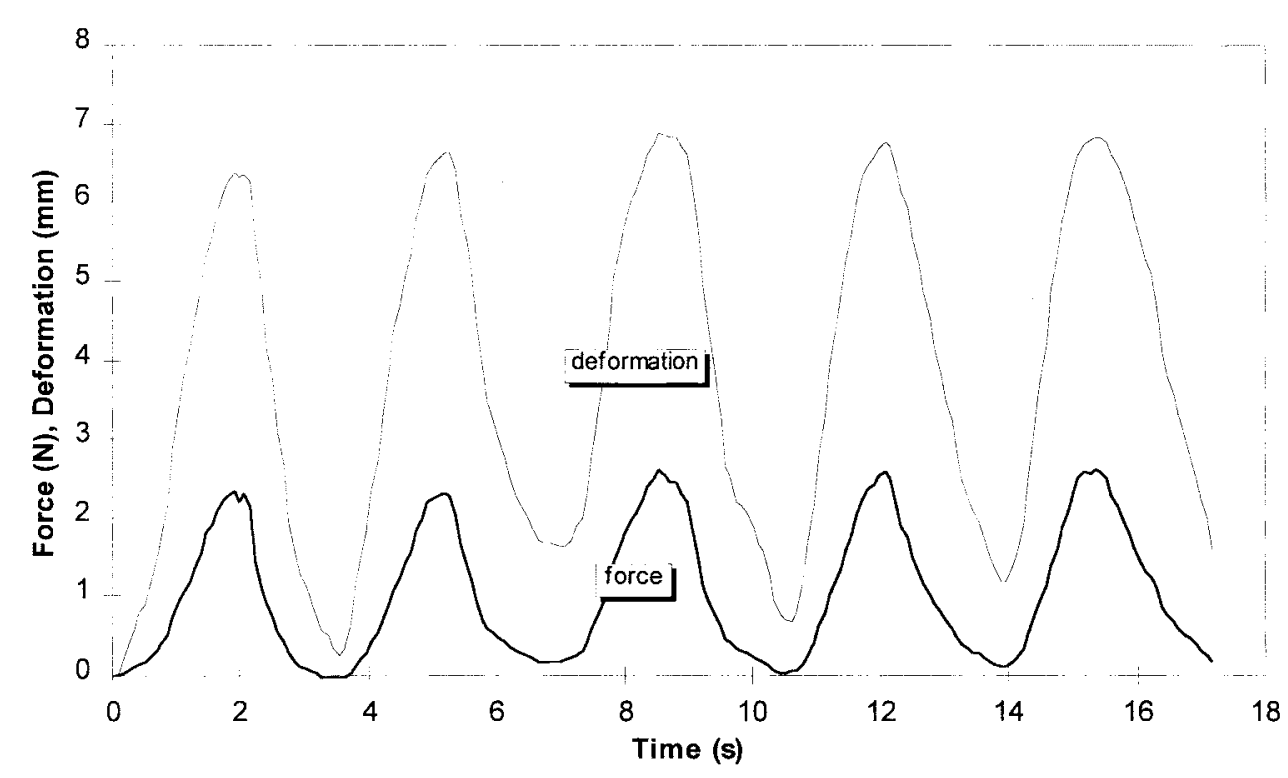

(a)

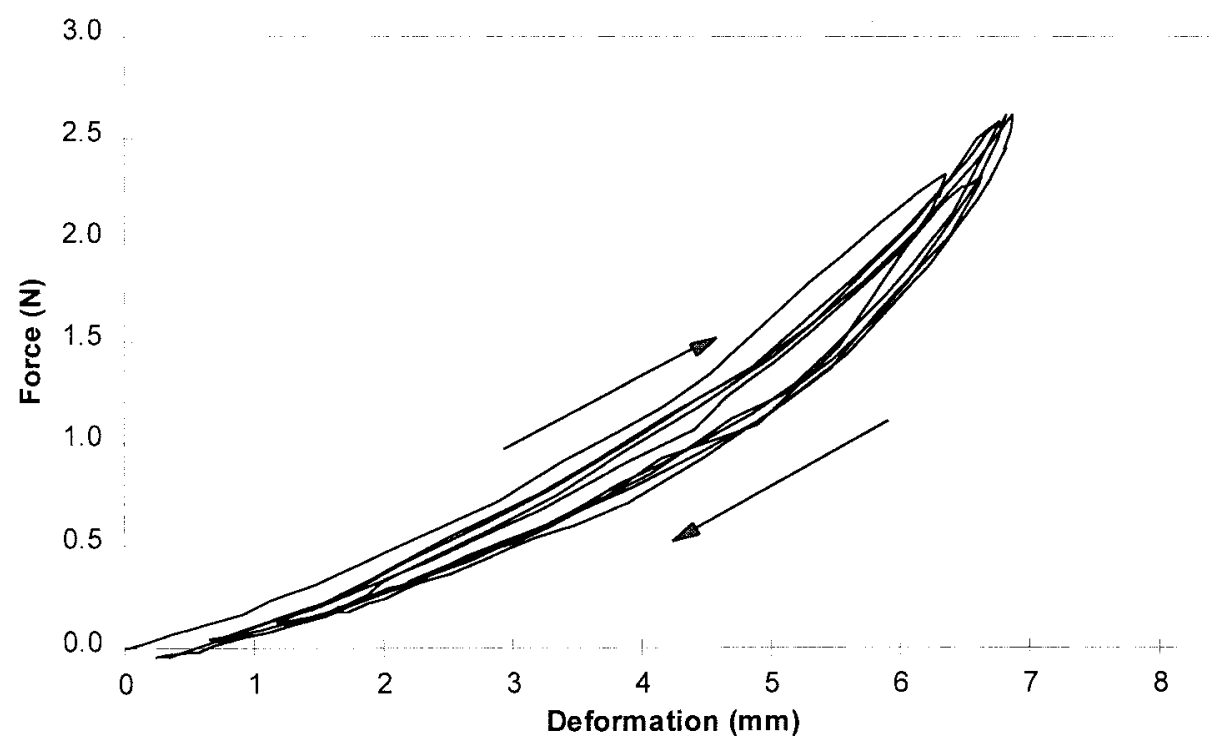

(b)

Fig. 4. A typical indentation response (a) and the corresponding load-indentation curve (b) recorded on the forearm of subject YZ with the hand relaxed. The original thickness of the soft tissue layer was $16.2 \mathrm{~mm}$.

value was found to be readily manageable for a hand-held test [33], [34]. Two hundred load-indentation data points were collected within a period of $17.2 \mathrm{~s}$. During a test, the maximum indentation was controlled within $30 \%$ of the initial thickness of the soft tissues, and the maximum indentation load was limited to $5 \mathrm{~N}$. It was noted that such indentation on the limb soft tissues at various sites with different thickness could be accepted by all of our subjects without pain. Ultrasound couplant gel was used to couple the ultrasound signal with the soft tissues.

During a test, the testing parameters of the indentor were manually controlled by the operator. The alignment of the probe was visually controlled by monitoring the amplitude of ultrasound reflection signals, which was found very sensitive to misalignment of the probe [33], [35]. The indentation rate, maximum indentation depth and maximum indentation load were also visually controlled by monitoring the indentation response which was shown on the computer monitor in real time.

Since the indentation with the current apparatus was relatively easy and quick to perform, as many as six trials were conducted at each site for each posture. Depending on the original thickness and stiffness of soft tissue at the testing site, each trial of indentation sequence would contain about 3-9 loading-unloading cycles. Fig. 4 shows a typical manual indentation response and the corresponding load-indentation curve. The trials with the maximum and minimum material parameter were excluded and the mean of the remaining four data sets was calculated as the result for this test. The influence on the results from the occasional movements of subjects 


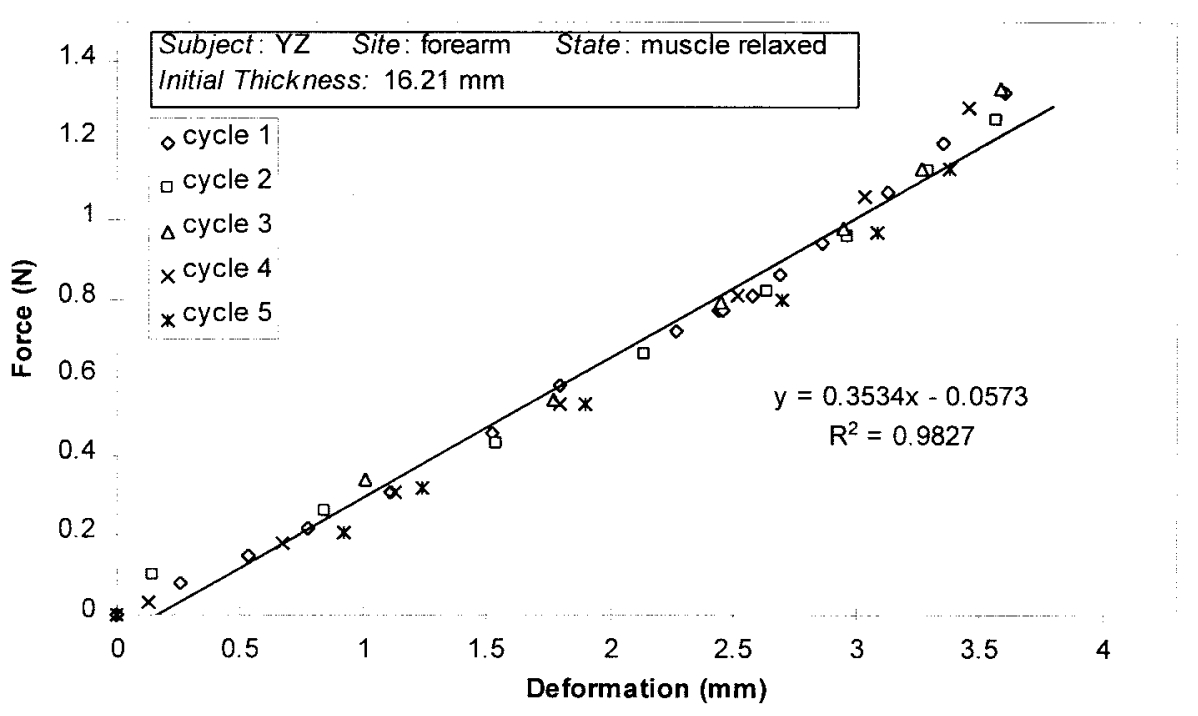

Fig. 5. The selected data points used for the estimation of the effective Young's modulus. Different symbols represented the data points of different cycles. The data in the loading phases with the indentation load larger than $0.5 \mathrm{~N}$ and smaller than $75 \%$ of the maximum value were selected. The plotted data was rescaled from raw data assuming the $0.5 \mathrm{~N}$ to be the reference state.

during each trial could be reduced by this process. Using the same testing protocol and data analysis method, a repeatability test was conducted on the left forearm of a normal young male subject [34]. Six tests with six trials for each test were performed on one site with an indentation rate around $4 \mathrm{~mm} / \mathrm{s}$.

\section{Data Reduction Methods}

A typical indentation response is shown in Fig. 4. This specific indentation test was composed of five loading-unloading cycles within a testing period of $17.2 \mathrm{~s}$. During the indentation test with the probe manually driven by hand, the indentation rate was difficult to control as a constant during a whole loading-unloading cycle. For this reason, the experimental data points in the loading phases with the indentation load larger than $0.5 \mathrm{~N}$ and smaller than $75 \%$ of the peak value were selected for the following data analysis. It was noted that the indentation rate within this range was relatively constant, and influences on the indentation response due to large variation of indentation rate near peak and valley in an indentation cycle could be reduced. The selected data points within such limits were plotted in Fig. 5 with different symbols representing the data points of different cycles. The plotted data was rescaled from the raw data assuming the $0.5 \mathrm{~N}$ to be the initial state. A linear regression was adopted to represent all the data points. The slope of the curve was the mean ratio of load to indentation, i.e., $P / w$, which reflects the biomechanical properties of the soft tissue. The small offset of the curve caused by the nonlinearity of the soft tissue mechanical properties was a result of the linear regression of the mildly nonlinear force-deformation data.

The effective Young's modulus was calculated using (1). This is a rigorous mathematical solution to the elastic indentation problem of a thin elastic layer bonded to a rigid half-space with a rigid, frictionless cylindrical plane-ended indentor [8]

$$
E=\frac{\left(1-\nu^{2}\right)}{2 a \kappa(\nu, a / h)} \frac{p}{w}
$$

where $h$ is the tissue thickness, and $\kappa$ is a scaling factor. This scaling factor provides a theoretical correction for the finite thickness of the elastic layer, and it depends on both the aspect ratio $a / h$ and Poisson's ratio $\nu$. The Poisson's ratio $\nu$ was assumed to be 0.45 in this study assuming soft tissue to be nearly an incompressible material. A similar assumption has been widely adopted in the literature [19], [25]-[28]. The radius of the indentor, i.e., the ultrasound transducer, was 4.5 $\mathrm{mm}$. With these constants, (1) can be written as the form

$$
E=\frac{1}{K(h)} \cdot \frac{P}{w}
$$

where $K(h)$ with a unit of length is a factor only depending on the thickness $h$ of the soft tissue if a constant Poisson's ratio $\nu$ is assumed. Using the value of $\kappa(a / h, \nu)$ proposed by Hayes et al. [8], the relationship between $K(h)$ and thickness $h$ can be derived. The curves under a Poisson's ratio of 0.3 , 0.45 , and 0.5 were plotted in Fig. 6. $K(h)$ can be interpolated for an arbitrary value of $h$. For the particular test shown in Figs. 4 and 5, the initial thickness $h$ was determined as 16.2 $\mathrm{mm}, K(h)$ was calculated as $15.6(\mathrm{~mm})$, and the average ratio of the applied load to the indentation depth $P / w$ was derived as $0.35 \mathrm{~N} / \mathrm{mm}$, the modulus could then be obtained as 22.6 $\mathrm{kPa}$ by using (2).

In this study, the limb soft tissue was assumed to be a linear elastic material, the nonlinear and viscoelastic properties were not taken into account in the present data analysis. The extracted modulus was named the effective Young's modulus.

\section{RESULTS}

\section{A. Validation Results}

Fig. 7 shows the results of the validation experiments for the new testing system performed on the porcine tissue. The data read from the material testing machine and those determined using the ultrasound indentation apparatus agreed very well. 


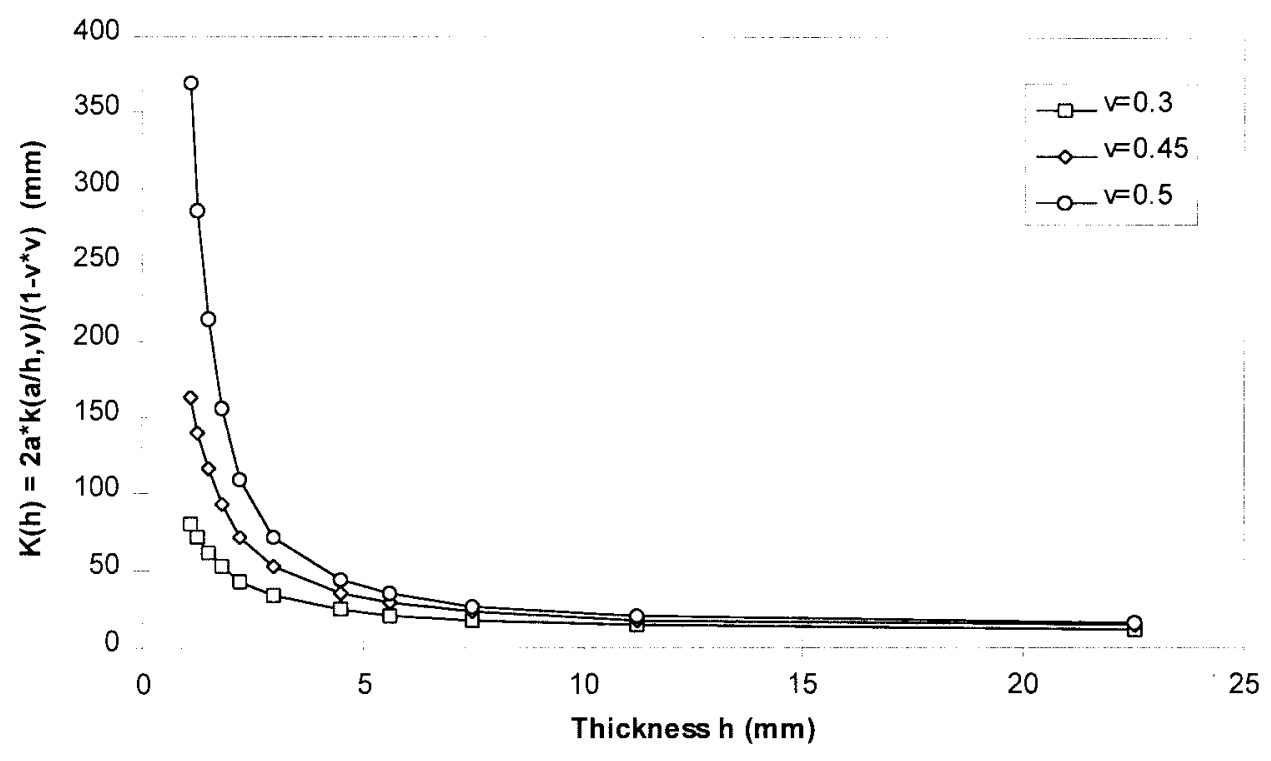

Fig. 6. The relationship between function $K(h)$ and thickness $h$ for the Poisson's ratio of $0.3,0.45$, and 0.5 .

If the values of force and displacement read from the material testing machine were assumed as real values, the variation of the force and displacement determined using the new system were within 3 and $0.3 \%$ of those real values, respectively.

\section{B. Soft Tissue Thickness}

In the repeatability test on the forearm, the result of the soft tissue thickness measurement was $17.26 \pm 0.13 \mathrm{~mm}$. The percentage standard deviation was calculated as $0.75 \%$. The original thickness of the lower limb soft tissues of the eight subjects at the four testing sites was in the range of $9.8 \pm 2.3$ $\mathrm{mm}$ (site 1, posture B) to $54.1 \pm 4.4 \mathrm{~mm}$ (site 4 , posture A). Fig. 8 shows slight variations of the soft tissue thickness with different body postures at the four testing sites. Paired $t$-tests demonstrated a highly significant change $(p<0.005)$ in the soft tissue thickness between posture $\mathrm{A}$ and $\mathrm{C}$, as well as between posture $\mathrm{B}$ and $\mathrm{C}$ at site 1 , site 2 and site 4 . A significant difference $(p<0.05)$ was also observed by paired comparison between postures A and B at site 1 , and between postures $\mathrm{B}$ and $\mathrm{C}$ at site 3 , although their variation of average value was small as shown in Fig. 8. No significant difference was observed between postures $\mathrm{A}$ and $\mathrm{B}$ at site 2 , site 3 and site 4. As the posture changed from sitting (posture A) to standing (posture $\mathrm{C}$ ), the mean tissue thickness of eight subjects increased $10.4 \%(1.1 \mathrm{~mm})$ at site 1 , and decreased $9.0 \%(3.6 \mathrm{~mm}), 3.8 \%(1.1 \mathrm{~mm})$ and $5.5 \%(3.0 \mathrm{~mm})$ at site 2 , site 3 and site 4 , respectively.

\section{Effective Young's Modulus}

In the repeatability test on the forearm, the result of the effective Young's modulus determination was $19.46 \pm 0.54$ $\mathrm{kPa}$. The percentage standard deviation was calculated as $2.8 \%$. The determined effective Young's modulus of lower limb soft tissues was in the range from $10.4 \mathrm{kPa}$ (subject FEN, site 1, posture A) to $89.2 \mathrm{kPa}$ (subject STE, site 3, posture $\mathrm{B})$, and it depended on site, posture, subject and gender. The standard deviations of eight subjects were calculated for each of the four sites and with each of the three postures, and were used to represent the subject variation. The subject variation of the effective elastic modulus with this definition ranged from 23.4 to $42.7 \%$.

Fig. 9 shows the variation of the effective Young's modulus due to different body postures at the four testing sites. Statistically significant differences $(p<0.05)$ of the effective Young's modulus of the soft tissue at the four sites were observed in all three postures. At the sites over the proximal medial tibia (site 1), lateral fibula (site 3) and calf area (site 4), the soft tissues became significantly stiffer when the posture changed from sitting with the foot relaxed (posture A) to sitting with the foot dorsiflexed (posture B). Almost no effect was observed due to such a postural change at site 2, which was located over the lateral femur. This result agreed with the concept that the underlying muscles at this site were only slightly involved in the action of foot dorsiflexion. When the posture changed from sitting (postures A and B) to standing (posture C), the stiffness of the soft tissue over the proximal medial tibia (site 1) decreased significantly, and those over the lateral femur (site 2), the lateral fibula (site 3) and the calf area (site 4) increased significantly. The soft tissue at the calf area (site 4) became dramatically stiffer when posture changed from sitting to standing; the mean effective Young's modulus changed from $15.5 \pm 4.3 \mathrm{kPa}$ to $51.3 \pm 12.5 \mathrm{kPa}$ for all eight subjects.

Fig. 10 shows that the effective Young's modulus of male subjects was significantly larger than that of female subjects in posture A. Similar results were obtained for the other two body postures. The mean modulus of limb soft tissues of the four female subjects ranged from 12.4 to $59.7 \mathrm{kPa}$ for the four sites with the three postures, and that of the four male subjects ranged from 18.6 to $70.2 \mathrm{kPa}$. The overall mean modulus of male subjects was $40 \%$ larger than that of female subjects.

Fig. 11 shows that there existed some correlations between the effective Young's modulus and the original thickness of soft tissues of the eight subjects with posture A (sitting) at 


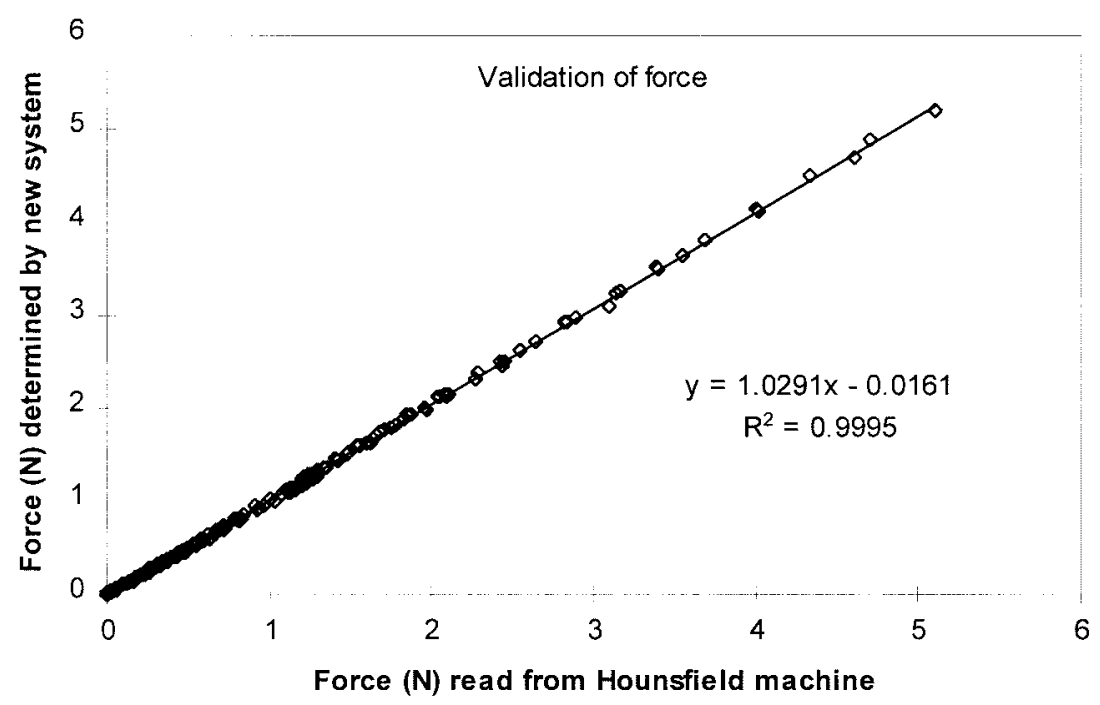

(a)

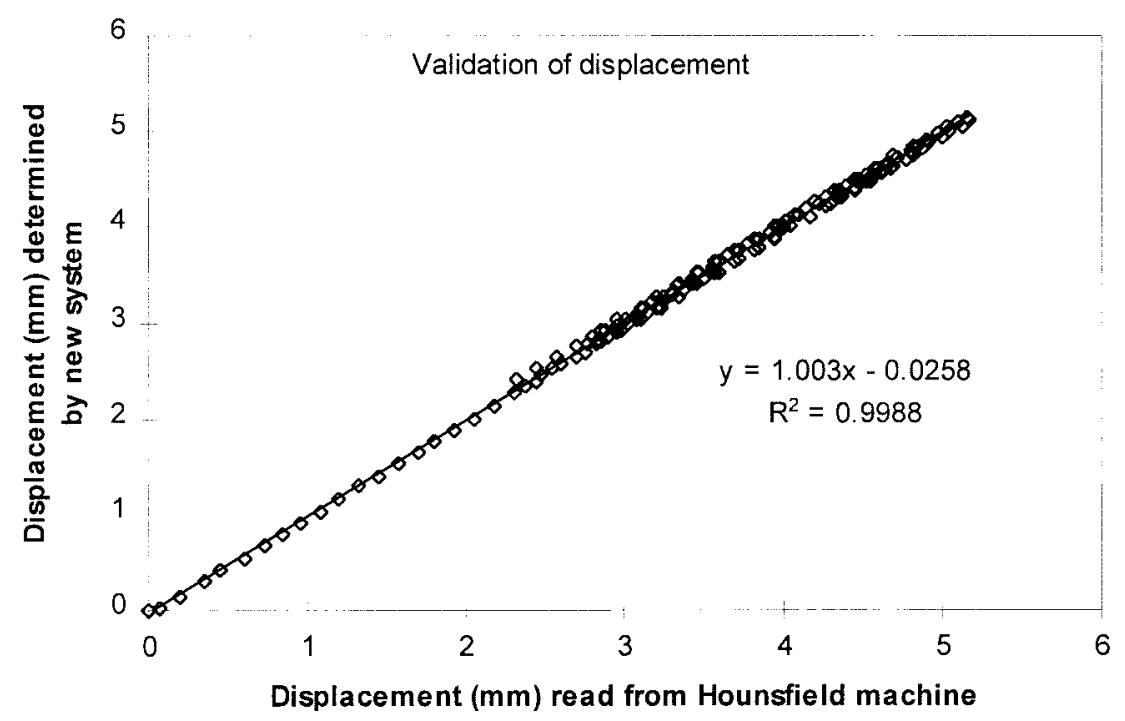

(b)

Fig. 7. Validation of the force (a) and deformation (b) determined using the indentation probe with those read from the material testing machine.

testing sites 1, 2, and 4. However, the determination coefficients $\left(R^{2}\right)$ of linear regression for all four sites were smaller than 0.3. Similar results were also obtained for postures B and C. Fig. 12 shows a tendency of obverse and reverse dependence between the change of modulus and the change of tissue thickness at site 3 and site 4, respectively, when the posture was changed from sitting (posture A) to standing (posture C). Meanwhile, the determination coefficients $\left(R^{2}\right)$ of linear regression for site 1 and site 2 were smaller than 0.1 . No significant correlations between modulus of soft tissue and its original thickness and between their changes induced by the alternation of postures could be established from the results obtained in this study.

\section{DISCUSSION}

Because of the lack of an easy-to-use quantitative testing method, comparisons of biomechanical properties of limb soft tissues at different sites with different body postures is rather lacking in the literature. Using an ultrasound indentation appa- ratus with a pen-size probe which was previously developed by the authors, indentation tests were conducted on eight normal young subjects. Lower limb soft tissues at four sites with three body postures were studied. A linear elastic indentation solution was used to extract the effective Young's modulus from indentation responses.

The determined effective Young's modulus ranged from 10.4 to $89.2 \mathrm{kPa}$, which was within a similar range as that reported in the literature: $60 \mathrm{kPa}$ [26], 53 to $141 \mathrm{kPa}$ [15], 50 to $145 \mathrm{kPa}$ [21], 27 to $106 \mathrm{kPa}$ [27], 21 to $194 \mathrm{kPa}$ [19]. These values varied among subjects, with pathological conditions, with sites and with states of the muscular activities. Results from this study demonstrated that the effective Young's modulus of lower limb soft tissues depended significantly on testing site, body posture, and gender. The mean modulus of the four female subjects ranged from 12.4 to $59.7 \mathrm{kPa}$ for the four sites with three postures, and that of the four male subjects ranged from 18.6 to $70.2 \mathrm{kPa}$. The overall mean modulus of males was $40 \%$ larger than that of females. 


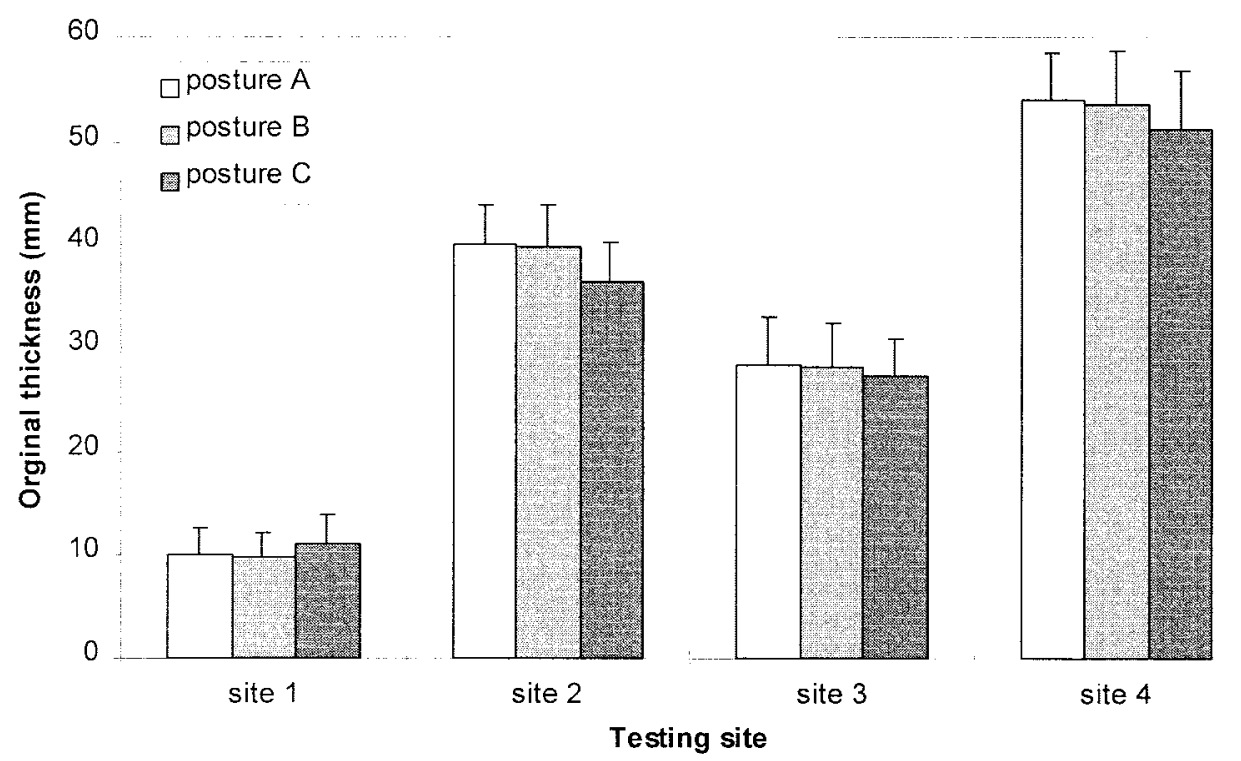

Fig. 8. The variation of the initial thickness of the soft tissue layers at the four testing sites of the eight subjects with three postures. The error bar represented the standard deviation of the data from the eight subjects.

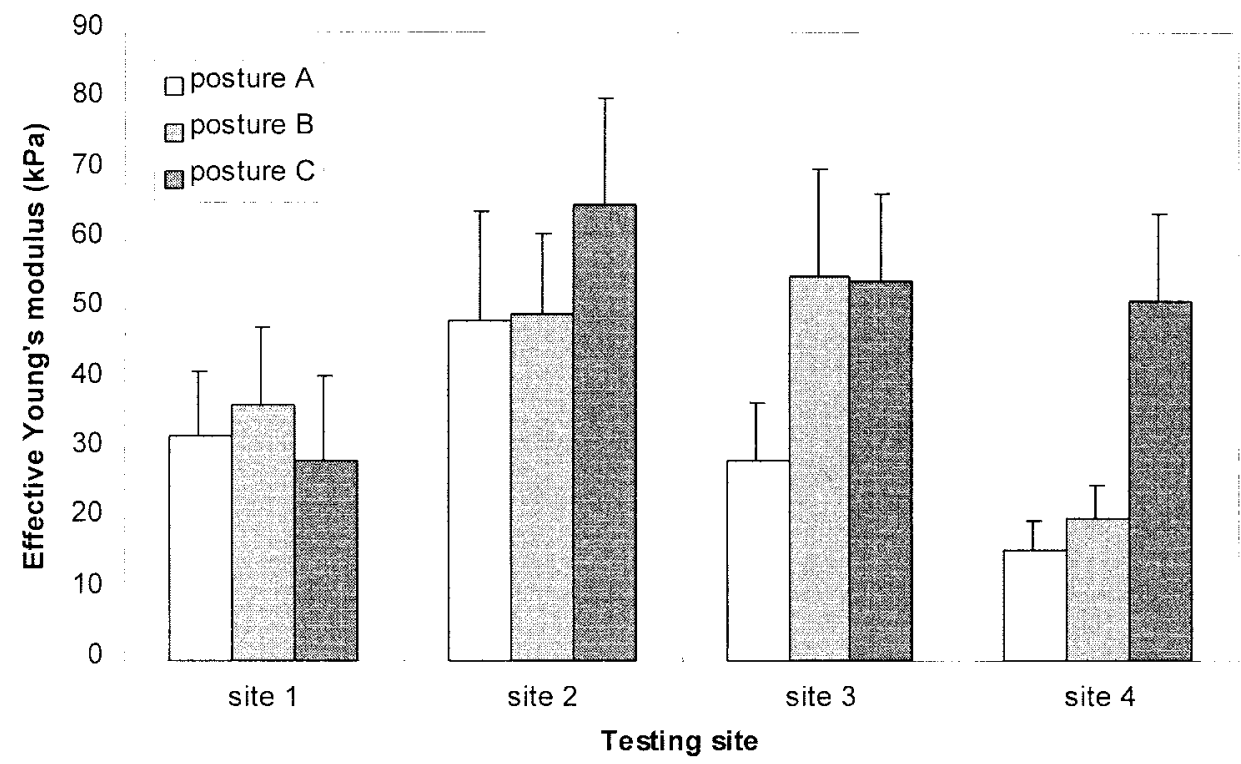

Fig. 9. The variation of the determined effective Young's modulus of the soft tissue layers at the four testing sites of the eight subjects with three postures. The error bar represented the standard deviation of the data from the eight subjects.

The original thickness of the lower limb soft tissues varied slightly (up to $10 \%$ ) with the change of body postures, compared with large variation of the modulus (up to 460\%). There existed some relationships between the effective Young's modulus and the original thickness of soft tissues, and between their relative changes induced by the alternation of postures. However, significant correlations were not established from the results of this study. The results confirmed that the activation states of the underlying muscles were the main reason for the variation of the determined effective Young's modulus of soft tissues due to the changes of body postures.

The above results were obtained from normal young subjects. Besides their significance on the investigation of biomechanical behavior of limb soft tissues, these results could also be used as a reference in lower limb prosthesis design, especially for the CAD/CAM prosthetic socket design and the FE analysis of prosthetic socket-residual limb interface problem. The body posture, site, and gender dependences of the effective Young's modulus of limb soft tissues have been reported in this paper for a narrow range of age. Further investigations on the age dependence and dependence of other variables of the effective Young's modulus are in planning. Additional studies on residual limb soft tissues of above-knee as well as belowknee amputees are currently being conducted at our Centre using the same approach as described in this paper.

The uncertainty of the Poisson's ratio of soft tissue would affect the extracted effective elastic properties. In the current study, a Poisson's ratio of 0.45 was assumed for the soft 


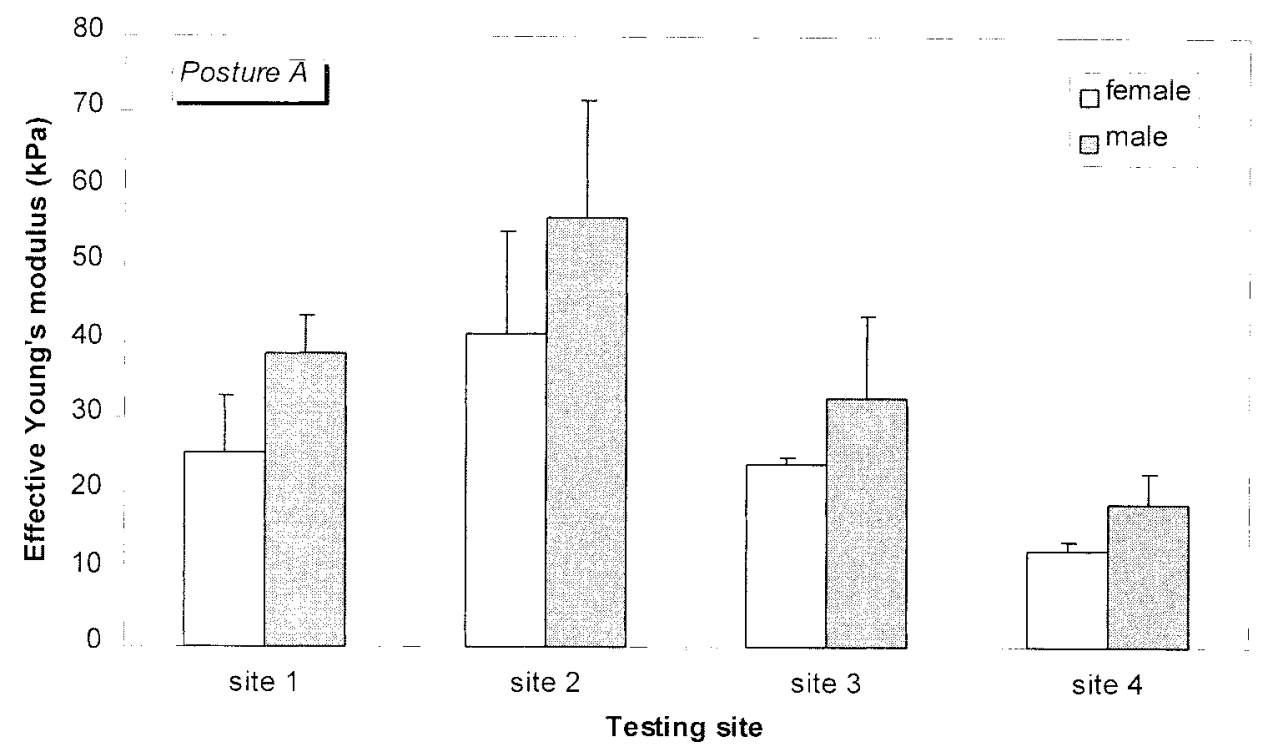

Fig. 10. The comparison of the determined effective Young's modulus between the female and male subjects. The error bar represented the standard deviation of the data from the four female and male subjects, respectively.

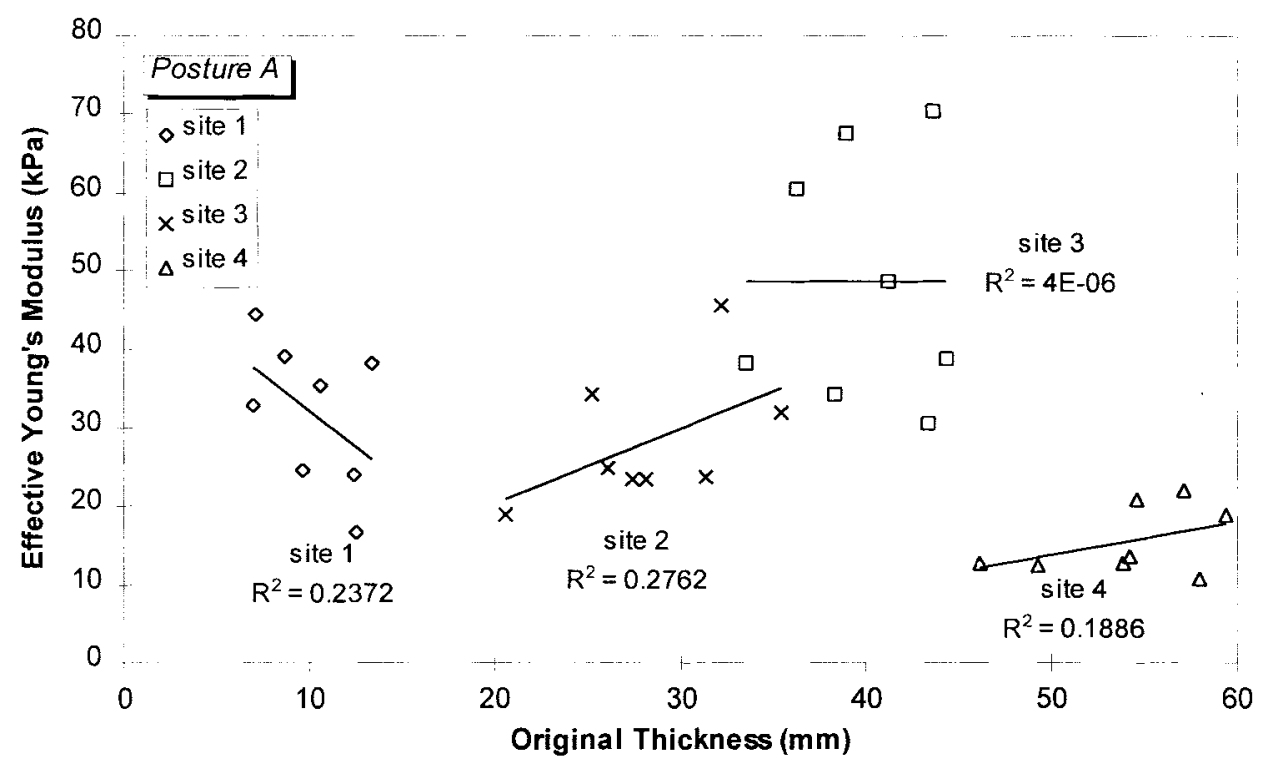

Fig. 11. The relationship between the determined effective Young's modulus and the original tissue thickness for the four testing sites with posture A (sitting). The lines were linear regressions for the four sets of data. $R^{2}$ was the determination coefficient of the linear regression.

tissue in the calculation, and the indentor diameter used was $9 \mathrm{~mm}$. If the Poisson's ratio is assumed to be 0.3 instead of 0.45 , the extracted modulus will increase by $14.1 \%$ to $19.4 \%$ as the tissue thickness changes from infinite to 20 $\mathrm{mm}$. While, if the Poisson's ratio is assumed to be 0.5 instead of 0.45 , the extracted modulus will decrease by 6.0 to $8.6 \%$ as the tissue thickness changes from infinite to $20 \mathrm{~mm}$. This effect would become more serious when the tissue thickness decreased, especially when the soft tissue was thinner than $5 \mathrm{~mm}$. In most indentation studies on skin and subcutaneous tissues, investigators have assigned a Poisson's ratio within the range from 0.45 to 0.5 to simulate the nearly incompressible behavior of the limb soft tissues as a whole during a dynamic situation [9], [19], [21], [25]-[27]. The absolute value of the extracted effective modulus may be affected by the assumed value of Poisson's ratio if the variation is large and the tissue thickness/indentor diameter ratio is small. Up to this point, it remains a challenge to clinically measure the Poisson's ratio of soft tissues independently.

The geometry of the limb soft tissues may be another source of uncertainty for the determined Young's modulus. Since the indentation solution [see (1)] employed in this study was derived for an infinite layer on a rigid substratum, the curvature of the limb surface and the limited dimension of the limb bone may affect the accuracy of the determined Young's modulus. Selection of a smaller indentor can reduce such effects. However, too small an indentor will limit the indentation to the superficial layer, and the determined Young's modulus will 


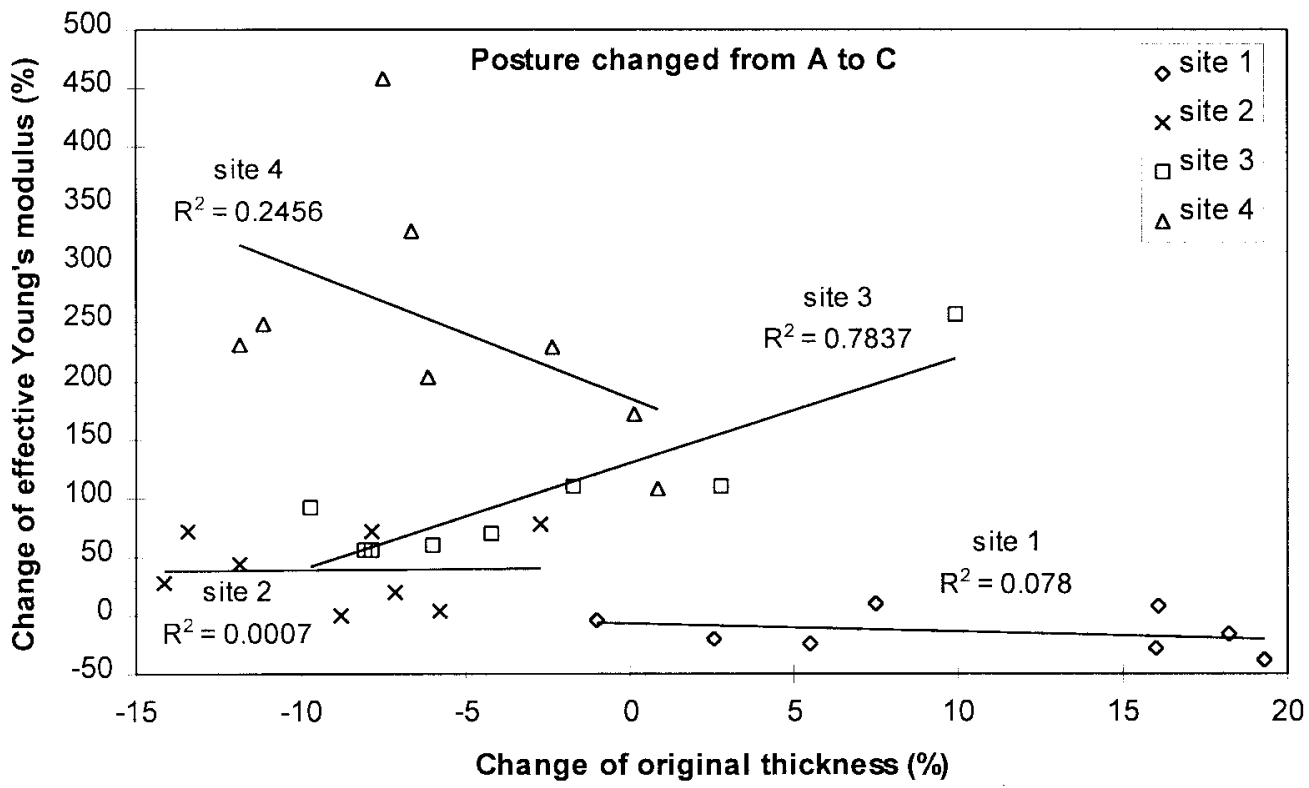

Fig. 12. The relationship between the change of the determined effective Young's modulus and the change of tissue thickness for the four testing sites with posture changed from posture A (sitting) to posture $\mathrm{C}$ (standing). The lines were linear regressions for the four sets of data. $R^{2}$ was the determination coefficient of the linear regression.

only represent the material property of that layer instead of the entire soft tissue layer. Numerical and experimental studies on the effects of the curvature of the limb surface as well as the size and shape of the underlying bone should be useful. Recent studies demonstrated that finite element analysis could be used to extract the material parameters of limb soft tissues from indentation responses [21], [25], [26], [29]. The limb geometry could be taken into account in the FE model [29]. If the processing time could be reduced for extensive application, FE analysis may be an alternative way to extract material parameters with higher accuracy.

In the indentation tests on skin and subcutaneous tissues, the indentor was conventionally aligned perpendicular to the skin surface. Misalignment of the indentor, however, is difficult to avoid, particularly when the indentation is performed manually. For linear elastic materials, the result of finite element analysis showed that although the stress distribution was influenced by the misalignment significantly, the total resultant force for the indentation was only slightly affected for up to eight degrees indentor misalignment [11]. In vitro experiments demonstrated that the indentation responses could be influenced by the misalignment of the indentor, especially at the sites with soft tissue thickness smaller than the diameter of the indentor [35]. It was observed with up to $12.5^{\circ}$ misalignment of the indentor, the effect on the indentation response decreased as the thickness increased, and became almost negligible when the tissue thickness became larger than double of the indentor diameter. The effects due to misalignment could also be reduced by adopting some amount of preload. This was one of the reasons for the exclusion of the indentation data with an applied load smaller than $0.5 \mathrm{~N}$. Residual limb soft tissues are also preloaded significantly due to socket confinement. During the test, the alignment of the probe could be visually controlled by monitoring the amplitude of the ultrasound reflection signals, capitalizing on the fact that the amplitude of the echo but not its time flight was very sensitive to the misalignment of the probe [35].

It is known that the mechanical properties of limb soft tissues are generally nonlinear and viscoelastic. This could be observed to some degree in the load-indentation response presented in Fig. 4. The nonlinearity and viscoelasticity were reduced in the data analysis used in this paper by excluding the data points near the peak and valley of the indentation cycles. For this reason, the determined Young's modulus was an effective modulus of the soft tissues for a range of deformation or applied load. Although such a simplified material parameter was not enough to describe the complex mechanical properties of soft tissues, it would still be very useful clinically. The results of FE analyzes demonstrated that the pressures applied to $70 \%$ of the interface area were between the range from 8 to $80 \mathrm{kPa}$ [30]. The material parameter obtained in this study was extracted with a similar range of applied pressure. Such a simple and easy-to-obtain material parameter may be particularly useful in the CAD/CAM design system for prosthetic sockets and cushions, where currently no quantitative material properties are involved in the design process. On the other hand, more precise descriptions of biomechanical properties of soft tissues are also desired in many applications, such as for the more realistic modeling of the socket-stump interface. For those purposes, two other approaches for extraction of the nonlinearity and quasilinear viscoelastic properties of soft tissues from the indentation results have also been proposed [32], [33].

\section{REFERENCES}

[1] D. L. Bader and P. Bowker, "Mechanical characteristics of skin and underlying tissues in vivo," Biomater., vol. 4, pp. 305-308, 1983.

[2] D. A. Boone, J. S. Harlan, and E. M. Burgess, "Automated fabrication of mobility aids: Review of the AFMA process and VA/Seattle ShapeMaker software design," J. Rehab. Res. Dev., vol. 31, pp. 42-49, 1994. 
[3] C. H. Daly and J. B. Wheeler III, "The use of ultrasonic thickness measurement in the clinical evaluation of the oral soft tissues," Int. Dent. J., vol. 21, pp. 418-429, 1971.

[4] D. Dean and C. G. Saunders, "A software package for design and manufacture of prosthetic sockets for transtibial amputees," IEEE Trans. Biomed. Eng., vol. 32, pp. 257-262, 1985.

[5] S. Dikstein and A. Hartzshtark, "In vivo measurement of some elastic properties of human skin," in Bioengineering and Skin, R. Marks and P. A. Payne, Eds. Lancaster, PA: MTP Press, 1981, pp. 45-53.

[6] M. Ferguson-Pell, S. Hagisawa, and R. D. Masiello, "A skin indentation system using a pneumatic bellows," J. Rehab. Res. Dev., vol. 31, pp. $15-19,1994$.

[7] S. A. Goss, R. L. Johnston, and F. Dunn, "Compilation of empirical ultrasonic properties of mammalian tissue," J. Acoust. Soc. Amer., vol. 68, pp. 93-108, 1980.

[8] W. C. Hayes, L. M. Keer, G. Herrmann, and L. F. Mockros, "A mathematical analysis for indentation tests of articular cartilage," $J$. Biomechan., vol. 5, pp. 541-551, 1972.

[9] M. Horikawa, S. Ebihara, F. Sakai, and M. Akiyama, "Non-invasive measurement method for hardness in muscular tissues," Med. Biol. Eng. Comput., vol. 31, pp. 623-627, 1993

[10] V. L. Houston, E. M. Burges, D. S. Childress, H. R. Lehneis, C. P. Mason, M. A. Garbarini, K. P. LaBlance, D. A. Boone, R. B. Chan, J. H. Harlan, and M. D. Brncick, "Automated fabrication of mobility aids (AFMA): Below-knee CASD/CAM testing and evaluation program results." J. Rehab. Dev. Res., vol. 29, pp. 78-124, 1992.

[11] D. T. Huang and A. F. T. Mak, "A finite element analysis of indentation on a soft tissue layers-The effect of indentor misalignment and nonparallel tissue layer," in Proc. Int. Conf. Biomed. Eng., Hong Kong, 1994, pp. 397-400.

[12] R. M. Kenedi, T. Gibson, J. H. Evans, and J. C. Barbenel, "Tissue mechanics," Phys. Med. Biol., vol. 20, pp. 699-717, 1975.

[13] E. Kirk and S. A. Kvorning, "Quantitative measurements of the elastic properties of the skin and subcutaneous tissue in young and old individuals," J. Gerontol., vol. 4, pp. 273-284, 1949

[14] E. Kirk and M. Chieffi, "Variations with age in elasticity of skin and subcutaneous tissue in human individuals," J. Gerontol., vol. 17, pp. 373-380, 1962.

[15] T. A. Krouskop, A. L. Muilenberg, D. R. Dougherty, and D. J. Winningham, "Computer-aided design of a prosthetic socket for an above-knee amputee," J. Rehab. Res. Dev., vol. 24, pp. 31-38, 1987.

[16] W. L. Kydd, C. H. Daly, and J. B. Wheeler III, "The thickness measurement of masticatory mucosa in vivo," Int. Dent. J., vol. 21 , pp. 430-441, 1971.

[17] W. L. Kydd, C. H. Daly, and D. Nansen, "Variation in the response to mechanical stress of human soft tissues as related to age," J. Prosthet. Dent., vol. 32, pp. 493-500, 1974.

[18] H. E. Lewis, J. Mayer, and A. A. Pandiscio, "Recording skinfold calipers for the determination of subcutaneous edema," J. Lab. Clin. Med., vol 66, pp. 154-160, 1965

[19] A. F. T. Mak, G. H. W. Liu, and S. Y. Lee, "Biomechanical assessment of below-knee residual limb tissue," J. Rehab. Res. Dev., vol. 31, pp. 188-198, 1994

[20] M. Malinauskas, T. A. Krouskop, and P. A. Barry, "Noninvasive measurement of the stiffness of tissue in the above-knee amputation limb," J. Rehab. Res. Dev., vol. 26, pp. 45-52, 1989.

[21] D. Reynolds, "Shape design and interface load analysis for below-knee prosthetic sockets," Ph.D. Dissertation, Univ. of London, England, 1988.

[22] J. E. Sanders, "Ambulation with a prosthetic limb: Mechanical stress in amputated limb tissues," Ph.D. Dissertation, Univ. Washington, Seattle, 1991.

[23] C. G. Saunders, J. Foort, M. Bannon, D. Dean, and L. Panych, "Computer-aided design of prosthetic sockets for below-knee amputees," Prosthet. Orthot. Int., vol. 9, pp. 17-22, 1985.

[24] H. Schade, "Untersuchungen zur organfunction des bindegewebes," Ztschr. f. Exper. Path. u. Therapis, vol. 11, pp. 369-399, 1912.

[25] M. B. Silver-Thorn, "Prediction and experimental verification of residual limb/prosthetic socket interface pressures for below-knee amputees," Ph.D. Dissertation, Northwestern Univ., Evanston, IL, 1991.

[26] J. W. Steege, D. S. Schnur, and D. S. Childress, "Prediction of pressure at the below-knee socket interface by finite element analysis," in Proc. Symp. Biomech. Normal Pathol. Gait, Boston, MA, AMSE, WAM, 1987, pp. 39-43.
[27] R. Torres-Moreno, "Biomechanical analysis of the interation between the above-knee residual limb and the prosthetic socket," Ph.D. Dissertation, Univ. Strathclyde, Glasgow, U.K., 1991.

[28] W. M. Vannah and D. S. Childress, "An investigation of the threedimensional mechanical response of bulk muscular tissue: Experimental methods and results," in Computational Methods in Bioengineering, R. L. Spilker and B. R. Simon, Eds., 1988, pp. 493-503.

[29] _ "Indentor tests and finite element modeling of bulk muscular tissue in vivo," J. Rehab. Res. Dev., vol. 33, pp. 239-252, 1996.

[30] M. Zhang, "Biomechanics of the Residual limb and prosthetic socket interface in below-knee amputees," Ph.D. Dissertation, Univ. London, England, 1995.

[31] Y. P. Zheng and A. F. T. Mak, "Development of an ultrasound indentation system for biomechanical properties assessment of soft tissues in-vivo," in Proc. 17th Annu. Int. Conf. IEEE Eng. Med. Biol. Soc., Montreal, P.Q., Canada, 1995, pp. 1599-1600.

[32] _ _ "An ultrasound indentation system for biomechanical properties assessment of soft tissues in-vivo," IEEE Trans. Biomed. Eng., vol. 43, pp. 912-918, 1996

[33] Y. P. Zheng, "Development of an ultrasound indentation system for biomechanical properties assessment of limb soft tissues in vivo," Ph.D Dissertation, Hong Kong Polytech. Univ., 1997.

[34] Y. P. Zheng and A. F. T. Mak, "Extraction of effective Young's modulus of skin and subcutaneous tissues from manual indentation data," in Proc. 19th Annu. Int. Conf. IEEE Eng. Med. Biol. Soc., Chicago, IL, 1997, pp. 2246-2249.

[35] Y. P. Zheng, D. T. Huang, and A. F. T. Mak, "Extraction of effective Young's modulus of skin and subcutaneous tissues from manual indentation data," in Proc. 19th Annu. Int. Conf. IEEE Eng. Med. Biol. Soc., Chicago, IL, 1997, pp. 2250-2253.

[36] J. C. Ziegert and J. L. Lewis, "In-vivo mechanical properties of soft tissue covering bony prominences," J. Biomechan. Eng., vol. 100, pp. 194-201, 1978

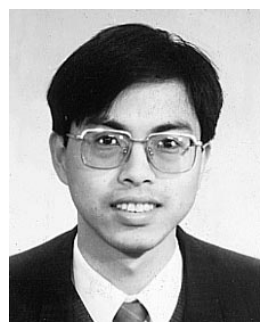

Yongping Zheng (S'95-M'97) received the B.Sc and M.Sc. degrees in electronics and electrical engineering from the University of Science and Technology of China, China, in 1990 and 1993, respectively. He received the $\mathrm{Ph} . \mathrm{D}$. degree in biomedical engineering from the Hong Kong Polytechnic University, China, in 1997.

After a Postdoctoral fellowship at the University of Windsor, Canada, he returned to the Hong Kong Polytechnic University in 1998. His main research interests include tissue biomechanics, acoustic microscope, nonlinear acoustics, and ultrasound instrumentation in bioengineering and in nondestructive testing.

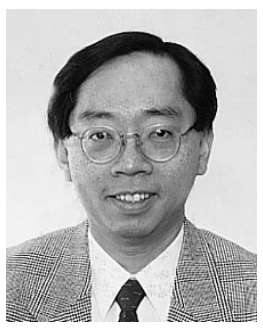

Arthur F. T. Mak (M'97) received the B.Sc. degree in engineering mechanics from the University of Illinois, Urbana, in 1976 and the Ph.D. degree in biomechanics from Northwestern University, Evanston, IL, in 1980.

After a Postdoctoral research associateship at the Rensselaer Polytechnic Institute, Troy, NY, he joined the Department of Bioengineering and Orthopaedic Surgery at the University of Pennsylvania Philadelphia, in 1983. He joined the Hong Kong Polytechnic University, China, in 1988 and became a full Professor in 1995. His main research interests include tissue mechanics, orthopaedic engineering, prosthetic biomechanics, pressure sore prevention, and other areas of rehabilitation engineering. 